\title{
Loss of purity by wave packet scattering at low energies
}

\author{
Jia Wang, C. K. Law and M.-C. Chu \\ Department of Physics, The Chinese University \\ of Hong Kong, Shatin, Hong Kong SAR, China
}

(Dated: August 14, 2018)

\begin{abstract}
We study the quantum entanglement produced by a head-on collision between two gaussian wave packets in three-dimensional space. By deriving the two-particle wave function modified by s-wave scattering amplitudes, we obtain an approximate analytic expression of the purity of an individual particle. The loss of purity provides an indicator of the degree of entanglement. In the case the wave packets are narrow in momentum space, we show that the loss of purity is solely controlled by the ratio of the scattering cross section to the transverse area of the wave packets.
\end{abstract}

PACS numbers: 03.67.Mn, 34.50.-s 
In this Brief Report we describe the quantum entanglement generated by wave packet scattering in three-dimensional free space. Unlike one-dimensional problems studied previously by one of us [1], scattering in 3D involves wave functions with much richer state structures for entanglement. Recently, we have demonstrated some interesting features for low energy eigen-functions in trapped systems [2]. For unbounded systems, Tai and Kurizki have analyzed the increase of entropy in terms of the scattering matrix [3]. Their approach is based on a particular form of two-particle wave functions in which the corresponding Schmidt decomposition can be expressed in pure plane wave bases [3]. For general two-particle wave functions, however, particles may not be paired in plane wave modes. Therefore a complete analysis of scattering effects on entanglement production remains open for investigations. Here we address the problem in the low-energy regime. Assuming the interaction potential is isotropic and short-ranged, we can employ the s-wave approximation to obtain the scattering wave functions. Our task is to determine the loss of purity of an individual particle, which serves as a measure of entanglement in our system with pure two-particle states.

The system under investigation consists of two interacting particles of equal mass $m$ in free space. The Hamiltonian in terms of center of mass and relative coordinates is given by: $H=H_{c m}+H_{r e l}$ with

$$
\begin{aligned}
H_{c m} & =\frac{P^{2}}{2 M} \\
H_{r e l} & =\frac{p^{2}}{2 \mu}+V(r) .
\end{aligned}
$$

Here $M=2 m$ is the total mass and $\mu=m / 2$ is the reduced mass. For convenience, we will use the units with $\hbar=\mu=1$. We assume that the interaction potential $V(r)$ is isotropic and has a short range $b$ such that $V(r) \approx 0$ for $r>b$. Initially, the two particles are in the form of (disentangled) gaussian wave-packets, each having a width $\sigma_{0}$ in momentum space. Their initial positions and average momenta are $\pm \mathbf{r}_{0}$ and $\mp \mathbf{k}_{0}$ respectively. The direction of $\mathbf{k}_{0}$ is chosen such that the packets make a head-on collision at later time (Fig. 1).

The initial two-particle wave function in momentum space is given by a product state: $\Phi\left(\mathbf{k}_{1}, \mathbf{k}_{2}, 0\right)=\phi_{1}\left(\mathbf{k}_{1}\right) \phi_{2}\left(\mathbf{k}_{2}\right)$, where

$$
\begin{gathered}
\phi_{1}\left(\mathbf{k}_{1}\right)=\Gamma\left(\mathbf{k}_{1}, \mathbf{k}_{0} ; \sqrt{2} / \sigma_{0}\right) e^{-i\left(\mathbf{k}_{1}-\mathbf{k}_{0}\right) \cdot \frac{\mathbf{r}_{0}}{2}} \\
\phi_{2}\left(\mathbf{k}_{2}\right)=\Gamma\left(\mathbf{k}_{2},-\mathbf{k}_{0} ; \sqrt{2} / \sigma_{0}\right) e^{i\left(\mathbf{k}_{2}+\mathbf{k}_{0}\right) \cdot \frac{\mathbf{r}_{0}}{2}}
\end{gathered}
$$

with $\Gamma(\mathbf{a}, \mathbf{b} ; c)$ being a gaussian function parameterized by the inverse width $c$ and the peak 


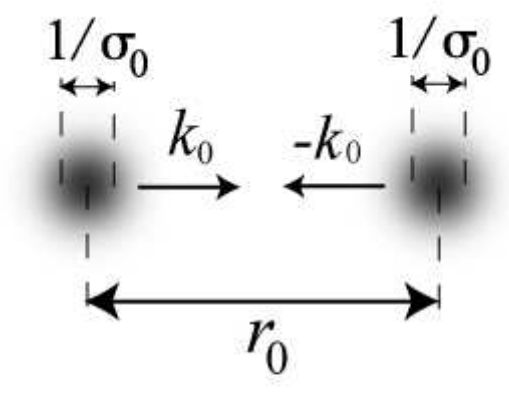

before

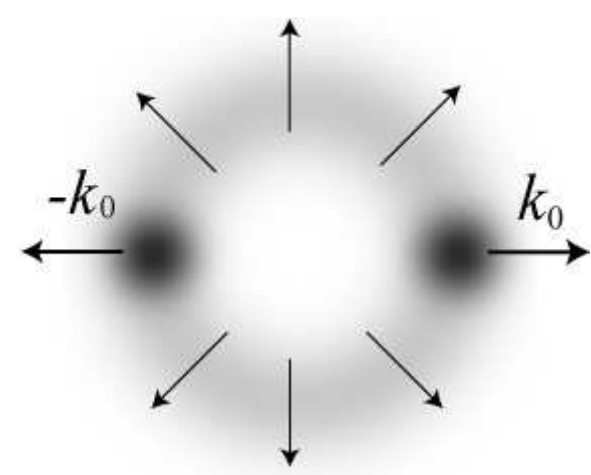

after

FIG. 1: An illustration of the system before and after a head-on collision of wave packets. Under the s-wave approximation, the scattered part of the single particle density (i.e., either particle 1 or particle 2) is a spherical shell shown in the right figure. The arrows indicate that the two particles go into opposite directions.

at $\mathbf{b}$,

$$
\Gamma(\mathbf{a}, \mathbf{b} ; c) \equiv\left(\frac{c^{2}}{2 \pi}\right)^{3 / 4} \exp \left[-\frac{c^{2}}{4}(\mathbf{a}-\mathbf{b})^{2}\right] .
$$

The function (5) allows us to express the wave packets in a compact form. After the scattering, the wave function in the long time limit takes the form:

$$
\Phi\left(\mathbf{k}_{1}, \mathbf{k}_{2}, t\right)=(N)^{-1 / 2}\left[\phi_{1}\left(\mathbf{k}_{1}\right) \phi_{2}\left(\mathbf{k}_{2}\right) e^{-i\left(k_{1}^{2}+k_{2}^{2}\right) t / 4}+\varepsilon \phi_{\text {scat }}\left(\mathbf{k}_{1}, \mathbf{k}_{2}, t\right)\right] .
$$

Here the first term corresponds to a non-scattering part that propagates freely, and the second term corresponds to the scattering part. The constants $N$ and $\varepsilon$ are normalization factors such that $\Phi$ and $\phi_{\text {scat }}$ are both normalized to unity. In this paper we treat $|\varepsilon| \ll 1$ as a small number.

To analyze the quantum entanglement, it is customary to study the entanglement entropy obtained from the Schmidt decomposition of (6). Quite generally, the Schmidt modes are not simply the momentum eigenfunctions, and the decomposition has to be performed numerically. We note that this is in contrast to the special case considered in Ref. 3], in which the Schmidt modes are momentum eigenfunctions. To gain insight of the problem analytically, we employ the purity function $\mathcal{P}$ as an alternative measure of entanglement. Such a function is defined by $\mathcal{P}=\operatorname{Tr}\left(\rho_{1}^{2}\right)$, where $\rho_{1}=\operatorname{Tr}_{2}\left(\rho_{12}\right)$ is the reduced density of 
the particle 1 , and $\rho_{12}$ corresponds to the two-particle density matrix associated with the state (6). For pure two-particle states considered in this paper, the smaller the value of $\mathcal{P}$, the higher the entanglement. A disentangled (product) state corresponds to $\mathcal{P}=1$. We remark that $\mathcal{P}$ shares similar features as entropy, but it has the key advantage that it is more accessible to theoretical analysis [4, 5, 6]. In atomic physics, $\mathcal{P}$ (or its inverse $\mathcal{P}^{-1}$ ) has also been employed to indicate the two-body correlations in various dynamical processes [4, 7].

Specifically, $\mathcal{P}$ takes an integral form in our system:

$$
\mathcal{P}=\iiint \int \Phi\left(\mathbf{k}_{1}, \mathbf{k}_{2}, t\right) \Phi\left(\mathbf{k}_{3}, \mathbf{k}_{4}, t\right) \Phi^{*}\left(\mathbf{k}_{1}, \mathbf{k}_{4}, t\right) \Phi^{*}\left(\mathbf{k}_{3}, \mathbf{k}_{2}, t\right) d^{3} \mathbf{k}_{1} d^{3} \mathbf{k}_{2} d^{3} \mathbf{k}_{3} d^{3} \mathbf{k}_{4} .
$$

From Eq. (4) and Eq. (6), we obtain the expression of $P$ up to the second order of $\varepsilon$ :

$$
\mathcal{P} \approx 1-2|\varepsilon|^{2}\left[1+I_{1}-I_{2}-I_{3}\right]
$$

where the integrals $I_{1}, I_{2}, I_{3}$ are defined by,

$$
\begin{aligned}
& I_{1}=\left|\iint \phi_{\text {scat }}^{*}\left(\mathbf{k}_{1}, \mathbf{k}_{2}, t\right) \phi_{1}\left(\mathbf{k}_{1}\right) \phi_{2}\left(\mathbf{k}_{2}\right) d^{3} \mathbf{k}_{1} d^{3} \mathbf{k}_{2}\right|^{2}, \\
& I_{2}=\int\left|\int \phi_{\text {scat }}^{*}\left(\mathbf{k}_{1}, \mathbf{k}_{2}, t\right) \phi_{1}\left(\mathbf{k}_{1}\right) d^{3} \mathbf{k}_{1}\right|^{2} d^{3} \mathbf{k}_{2} \\
& I_{3}=\int\left|\int \phi_{\text {scat }}^{*}\left(\mathbf{k}_{1}, \mathbf{k}_{2}, t\right) \phi_{2}\left(\mathbf{k}_{2}\right) d^{3} \mathbf{k}_{2}\right|^{2} d^{3} \mathbf{k}_{1} .
\end{aligned}
$$

These integrals describe the interference between a non-scattered wave and a scattered wave. It is interesting to note that there are no first order terms in $\varepsilon$ in Eq. (8), as these terms cancel each other once the $\varepsilon$ dependence in the normalization constant $N$ is taken into account. We also remark that as long as $\varepsilon$ is a small parameter, Eq. (8) is valid for general two-particle states that are initially separable, not just for gaussian wave packets.

To calculate $\varepsilon$ and $\phi_{\text {scat }}$, let us rewrite Eq. (6) in terms of center of mass and relative coordinates: $\Phi\left(\mathbf{k}_{1}, \mathbf{k}_{2}, t\right)=(N)^{-1 / 2} \phi_{c m}(\mathbf{K}, t) \phi_{r e l}(\mathbf{k}, t)$, where $\mathbf{K}=\mathbf{k}_{1}+\mathbf{k}_{2}, \mathbf{k}=\left(\mathbf{k}_{1}-\mathbf{k}_{2}\right) / 2$, $\phi_{c m}(\mathbf{K}, t)=\Gamma\left(\mathbf{K}, 0 ; 1 / \sigma_{0}\right) e^{-\frac{1}{8} K^{2} t}$. Since we are interested in low-energy scattering processes, we may keep only the s-waves of the scattered part in $\phi_{r e l}(\mathbf{k}, t)$, i.e.,

$$
\phi_{r e l}(\mathbf{k}, t) \approx \phi_{r e l}^{N S}(\mathbf{k}, t)+\varepsilon \eta^{(s)}(\mathbf{k}, t),
$$

where $\phi_{r e l}^{N S}(\mathbf{k}, t)=\phi_{r e l}(\mathbf{k}, 0) e^{-i \frac{1}{2} k^{2} t}$ is the freely propagating non-scattered part. In this way, we have $\phi_{\text {scat }}\left(\mathbf{k}_{1}, \mathbf{k}_{2}, t\right) \approx \phi_{c m}(\mathbf{K}, t) \eta^{(s)}(\mathbf{k}, t)$. 
Under the assumption that the two particles are well separated (initially and finally) [8], the scattering part $\varepsilon \eta^{(s)}(\mathbf{k}, t)$ is given by,

$$
\varepsilon \eta^{(s)}(\mathbf{k}, t)=\frac{1}{(2 \pi)^{3}} \iint \phi_{r e l}\left(\mathbf{k}^{\prime}, 0\right) f_{0}\left(k^{\prime}\right) \frac{e^{i k^{\prime} r}}{r} e^{-i \mathbf{k} \cdot \mathbf{r}-i k^{\prime 2} t / 2} d^{3} \mathbf{k}^{\prime} d^{3} \mathbf{r}
$$

where,

$$
f_{0}(k)=\frac{e^{i 2 \theta(k)}-1}{2 i k}
$$

is the s-wave scattering amplitude, and $\theta(k)$ is the s-wave scattering phase shift. After some calculations, we obtain,

$$
\varepsilon \eta^{(s)}(\mathbf{k}, t)=\frac{\sigma_{0}^{2}}{4 k_{0}-i 2 \sigma_{0}^{2} r_{0}}\left[e^{2 i \theta(k)}-1\right] \Gamma\left(\mathbf{k}, k_{0} \hat{\mathbf{k}} ; \frac{2}{\sigma_{0}}\right) \frac{e^{i\left(k-k_{0}\right) r_{0}}}{k} e^{-i \frac{1}{2} k^{2} t} .
$$

The constant $\varepsilon$ is determined from the norm of the right side of Eq. (15). We may Taylor expand $\theta(k)$ at $k_{0}$ to the second order. The normalization condition is a Gaussian integral that can be calculated explicitly. This gives

$$
|\varepsilon|^{2}=\frac{\sigma_{0}^{2}}{k_{0}^{2} \gamma^{2}}\left[1-\operatorname{Re}\left\{e^{2 i \theta\left(k_{0}\right)} \sqrt{\frac{2}{2-i \sigma_{0}^{2} \theta^{\prime \prime}\left(k_{0}\right)}} \exp \left[-\frac{\sigma_{0}^{2} \theta^{\prime}\left(k_{0}\right)^{2}}{2-i \sigma_{0}^{2} \theta^{\prime \prime}\left(k_{0}\right)}\right]\right\}\right]
$$

where $\theta^{\prime}\left(k_{0}\right)$ and $\theta^{\prime \prime}\left(k_{0}\right)$ are first and second derivatives of $\theta\left(k_{0}\right)$, and $\gamma^{2} \equiv 1+\left(\frac{\sigma_{0}^{2} r_{0}}{2 k_{0}}\right)^{2}$ in the denominator corresponds to the spreading factor of the spatial width of the packets (since $r_{0} / k_{0}$ is the time of collision). Therefore the spreading of the wave packets would decrease the norm of the scattered wave function $|\varepsilon|^{2}$ as expected. We also note that the value of the bracket $[1-\operatorname{Re}\{\ldots\}]$ is bounded between 0 and 2 , and therefore $|\varepsilon|^{2}$ is smaller than $2 \sigma_{0}^{2} / k_{0}^{2}$.

With the results of $\varepsilon$ and $\phi^{(s)}$, we find that $I_{1} \approx \frac{\sigma_{0}^{2}}{2 k_{0}^{2}}, I_{2} \approx \frac{2 \sigma_{0}^{2}}{3 k_{0}^{2}}, I_{3} \approx \frac{2 \sigma_{0}^{2}}{3 k_{0}^{2}}$ are all of the order of $\sigma_{0}^{2} / k_{0}^{2}$. Because of the prefactor $|\varepsilon|^{2}$ in Eq. (8), these integrals's contribution to $P$ is about $\sigma_{0}^{4} / k_{0}^{4}$, which will be neglected. Hence the purity of final state is approximately

$$
\mathcal{P} \approx 1-2|\varepsilon|^{2}
$$

where $|\varepsilon|^{2}$ is given by Eq. (16).

Further simplification of this result can be made in the limit $\sigma_{0}^{2} \theta^{\prime \prime}\left(k_{0}\right) \ll 1$, and $\sigma_{0} \theta^{\prime}\left(k_{0}\right) \ll 1$, i.e., the wave-packets are very narrow in the momentum space. In this limit, we have $|\varepsilon| \approx \sqrt{2}\left|f_{0}\left(k_{0}\right)\right| \sigma_{c}$, where $1 / \sigma_{c} \equiv \gamma / \sigma_{0}$ is the spatial width of the wave packets at the collision time. Alternatively, we may employ the scattering cross section $S_{0}\left(k_{0}\right)=4 \pi\left|f_{0}\left(k_{0}\right)\right|^{2}$, so that

$$
1-\mathcal{P} \approx 4 \sigma_{c}^{2}\left|f_{0}\left(k_{0}\right)\right|^{2}=\frac{\sigma_{c}^{2} S_{0}\left(k_{0}\right)}{\pi}
$$


Therefore the purity of the two-particle wave function after scattering can now be explicitly expressed in terms of the s-wave scattering cross section as well as the widths of wave packets. However, we remark that such a simple relation is valid if $\sigma_{0}$ is sufficiently small. The result can become more complicated when $\sigma_{0} \theta^{\prime}\left(k_{0}\right)$ or $\sigma_{0}^{2} \theta^{\prime \prime}\left(k_{0}\right)$ in Eq. (16) are not negligible.

Equations (16-18) are the main results of this paper. We see that the degree of entanglement (quantified by $1-\mathcal{P}$ ) is determined by a simple dimensionless parameter $\sigma_{c}^{2} S\left(k_{0}\right)$. Since $1 / \sigma_{c}$ is the spatial width of an individual wave packet at the collision time, $\sigma_{c}^{2} S\left(k_{0}\right)$ is just the ratio of scattering cross section to the characteristic cross area of the wave-packet in position space. Hence, a stronger entanglement can be generated for systems with a larger value of the ratio. For example, this can be achieved by exploiting resonance scattering in which $S\left(k_{0}\right)$ can be enhanced near the resonance energies defined by the interaction potential [1, [3].

We point out that the degree of entanglement is typically small. This is due to the fact that the two-particle wave function is dominated by an un-scattered part, which is a product state. However, if mainly the scattered part is observed (for example, by detecting directions different from the incident one), then the relevant wave functions can have a much higher degree of entanglement. For the s-wave function given in Eq. (15), if $\sigma_{0}$ is small such that the phase shift can be treated as a constant $\theta\left(k_{0}\right)$, then the normalized (relative coordinate) scattered wave function is a spherical shell of radius $k_{0}$ and thickness $\sigma_{0}$ in momentum space. We find that the corresponding purity function $\mathcal{P}$ has a leading term proportional to $\sigma_{0}^{2} / k_{0}^{2}$ when $\sigma_{0} / k_{0} \ll 1$ is a small parameter. Therefore the narrower the width of the wave packet, the stronger the entanglement in the scattered part of the wave function.

To conclude, we present a simple and general formula that approximates the loss of purity due to a head-on collision between two gaussian wave packets in three dimensional space. As long as the scattering is dominated by s-waves, our results provide a quantitative measure of quantum entanglement generated. In particular, our approach allows us to identify the key parameter $\sigma_{c}^{2} S\left(k_{0}\right)$, that explicitly connects the scattering cross section and the width of wave packets to the degree of quantum entanglement. 


\section{Acknowledgments}

This work is supported in part by the Research Grants Council of the Hong Kong Special Administrative Region, China (Project No. 400504).

[1] C. K. Law, Phys. Rev. A 70, 034101 (2004).

[2] Jia Wang, C. K. Law, and M.-C. Chu, Phys. Rev. A 72, 022346 (2005).

[3] A. Tal and G. Kurizki, Phys. Rev. Lett. 94, 160503 (2005).

[4] R. Grobe, K. Rzążewski and J .H. Eberly, J. Phys. B 27, L503 (1994).

[5] J. Gemmer, A. Otte, and G. Mahler, Phys. Rev. Lett. 86, 1927 (2001).

[6] Ph. Jacquod, Phys. Rev. Lett. 92, 150403 (2004).

[7] W.-C. Liu, J. H. Eberly, S. L. Haan and R. Grobe, Phys. Rev. Lett. 83, 520 (1999); R. E. Wagner, P. J. Peverly, Q. Su, and R. Grobe, Laser Phys. 11, 221 (2001); M. V. Fedorov, M. A. Efremov, A. E. Kazakov, K. W. Chan, C. K. Law, and J. H. Eberly, Phys. Rev. A 69, 052117 (2004).

[8] See for example, E. Merzbacher, Quantum Mechanics, second edition (Wiley, New York 1970). 